\title{
On Gravity-centric Cosmology and the Implications of a Universe Awash with Plasma
}

\author{
David B. Smith* \\ 6 Westport Avenue, Queenstown, South Australia 5014
}

\begin{abstract}
The Standard Model (of Cosmology) is the collective name given to the generally accepted explanation of the origin and current behavior of our Universe. It has at its heart the often quoted theory of 'The Big Bang' and is based upon Einstein's theory of General Relativity. More recently, theorists have attempted to integrate the known forces in the universe. For many decades there have been significant challenges to the Standard Model. Some scientists either feel uncomfortable with an emphasis on mathematics at the expense of empirical evidence, or they know of alternate theories to account for much of what we observe, some of which offer simpler explanations than the Standard Model. This paper is aimed at introducing readers to an alternative broadly known as the Plasma Universe or Plasma Cosmology, to examine some of the recent work of several theorists, and to highlight the importance of the role of plasma in space. It is deliberately broad in scope and sparse in detail to allow those from all disciplines to understand it without the need for dedicated training in any specific field, and to provoke questions that the inquisitive may wish to pursue further.
\end{abstract}

Keywords: Gravity, plasma cosmology, plasma universe, standard model, problems.

\section{INTRODUCTION}

Due to the need to render many high-quality images to illustrate some of the points made and to maintain the flow of text in this paper, supplementary images are supplied and referenced thus: [Supplement $\boldsymbol{x}$, Figure $\boldsymbol{y}$ ]. High-resolution copies are posted online at http://www.plasmaresources.com/supplement1.html.

Our science and technology awareness and abilities have grown exponentially throughout the last century, and the trend continues into this, the $21^{\text {st }}$. Tremendous amounts and newer types of data have been revealed due to our greatly enhanced ability to make observations. However, these newfound data have also brought to the attention of some, that many of the older and untestable scientific theories have, over time, quietly embedded themselves as scientific fact in our modern psyche. This has taken place to the point where these so-called 'facts' have been accepted and go unquestioned. One area of science where this is true is the study of how the universe works.

The Standard Model (of Cosmology) is the collective name given to the generally accepted explanation of the origin and current behavior of our Universe. It has at its heart the often quoted theory of 'The Big Bang' and is based upon Einstein's theory of General Relativity. More recently, theorists have attempted to integrate the known forces in the universe. For many decades there have been significant challenges to the Standard Model. Some scientists either feel uncomfortable with an emphasis on mathematics at the

*Address correspondence to this author at the 6 Westport Avenue, Queenstown, South Australia 5014; Tel: +618 8240 0284;

E-mails: davesmith_au@plasmaresources.com, davesmith_au@internode.on.net expense of empirical evidence, or they know of alternate theories to account for much of what we observe, some of which offer simpler explanations than the Standard Model. This paper is aimed at introducing readers to an alternative broadly known as the Plasma Universe or Plasma Cosmology, to examine some of the recent work of several theorists, and to highlight the importance of the role of plasma in space. It is deliberately broad in scope and sparse in detail to allow those from all disciplines to understand it without the need for dedicated training in any specific field, and to provoke questions that the inquisitive may wish to pursue further.

Plasma Cosmology is based on the work of Kristian Birkeland, Hannes Alfvén, Anthony Peratt and others, and requires an interdisciplinary approach. In particular the principles of plasma physics and electrical engineering are applied to astrophysics and cosmology, though there are other disciplines which may also be affected.

\subsection{SETTING THE SCENE}

Science is the art of taking a set of observations, proposing an explanatory structure of why and how those observations came about (hypothesis), and then rigorously testing that hypothesis to see if it comports with new observations. Hypotheses that repeatedly withstand such testing can be called theories. Where rigorous testing cannot be undertaken or new observations cannot be reconciled with the hypothesis, then what remains can only be described as a failed or falsified hypothesis - superficially plausible though it may seem. It is a fact that major "theories" of popular cosmology and its Standard Model have not been tested because they cannot be, so few if any actual facts or truths have been established. 
Real progress in the sciences can only be achieved through effective cooperation and honest answers to appropriate questions. We must also keep in mind that a measure of skepticism is a healthy thing to have, and we must be actively inquisitive and prepared to ask the awkward questions without bias towards any particular theory.

Here is something to ponder:

The gravity relied upon by the Standard Model is such a weak force that mainstream science has significant problems in adequately explaining how our universe really works!

Astrophysicists, theoretical mathematicians, astronomers and cosmologists, i.e. the people who have traditionally developed and supported the Standard Model, all have their own separate specialist areas which they focus upon, but they generally have one thing in common - they are not plasma scientists and they often have only limited training in electrical engineering principles.

The gravity-centric theories offered as explanations for the mysteries we observe in the universe and which underpin the Standard Model cannot be tested experimentally, though computer simulations flourish. On the other hand, plasma cosmology is based on sound plasma physics as developed in the laboratory and much of which has been tested both with experiments and in advanced plasma-based computer simulations. Due to better scientific capabilities and understanding today, a solid body of evidence supports credible alternative explanations for what we observe in space. This requires us to reconsider our assumptions.

\section{A FEW PROBLEMS WITH THE STANDARD MODEL}

Throughout the period when the Standard Model was being established, technological capabilities improved greatly, but some serious questions have also been raised. A few significant scientists faced up to these questions with professionalism and honesty:

"Big Bang cosmology refers to an epoch that cannot be reached by any form of astronomy, and, in more than two decades, it has not produced one single successful prediction" [1].

Many other leading scientists have remained conspicuous through their silence. Following are a few areas of the Standard Model with which some theorists have serious concerns:

(To aid the flow of information in this section, some of the specific problems identified have a plasma-based alternative presented within the section).

\subsection{Gravity as the Prime Mover}

Gravity is the weakest of the forces known to physicists. Even a fridge magnet can lift up a paperclip in defiance of the gravitational pull of the entire Earth. If we were to imagine the Sun as a speck of dust, the next nearest star would be another speck of dust around four miles away. If we consider the gravitational attraction between two grains of dust four miles apart, that is the miniscule force which the Standard Model relies upon to account for most of the workings of our universe.
The electrical force is 39 orders of magnitude greater than the gravitational force. Ignoring the role of electricity in the cosmos has led to the absurdity of gravity, and to some degree magnetism, being the only forces available to astrophysicists and cosmologists and the plethora of contradictory, unsubstantiated and non-falsifiable theories this situation has produced.

Of recent years, confirmation of ubiquitous magnetic fields throughout the cosmos has forced theorists to acknowledge their existence, yet they still largely ignore the electric currents which must be associated with these fields.

\subsection{The Big Bang}

An ever expanding universe and a faster-than-light expansion of the big bang event, arose from interpretation of observed changes in the light coming from distant galaxies. The Cosmic Microwave Background and distances based on redshift are offered in support of the big bang event [2].

Many plasma theorists see the big bang as both unnecessary and unsupported by empirical evidence. No counter theory to the big bang is offered and quite frankly this author finds it somewhat pretentious to be proffering explanations of how and when the universe 'came into' existence.

\subsection{The Cosmic Microwave Background}

The 'discovery' of the Cosmic Microwave Background (CMB) is usually attributed to two Bell Telephone Laboratories engineers who received unexpected 'radio static' via their radio telescope equipment and first published a paper on the phenomenon in 1964 [3]. However it had been detected in the late 1930s and interpreted as radiation excitation by Australian astronomer A. McKellar in 1941 [4].

The standard interpretation is that when the evenly distributed 'temperature' of the CMB which is measured at $2.7^{\circ} \mathrm{K}$, is taken together with its very smooth structure, and with the theory of redshift distances, the combination is said to provide significant support for the reality of the Big Bang event.

One of the problems with CMB theory is that IF it is the most distant thing we can see, (a remnant of the Big Bang) then we should observe the silhouettes of galaxy clusters and other major cosmic structures imposed on this image, which we do not [5].

Radio astronomy data now reveals that what astronomers call CMB radiation from the far edge of the visible universe, is actually likely to be electromagnetic noise occurring in our own cosmic neighborhood. Electric currents flowing in plasma naturally generate radio noise right across the spectrum, so the CMB could well be a type of local 'radio fog' [6, 7].

Where plasma double layers form in space 'radio noise' increases, thereby giving the appearance of a relative 'hot spot' which astronomers have tended to interpret in exotic ways, such as pulsars, mysterious x-ray sources, neutron stars, quasars, etc. but which are in fact quite understandable by considering the effects of plasma interaction. Electromagnetic hot spots 'strangely' match the pattern of measured temperature hot spots in the most detailed mappings of the CMB [7]. 


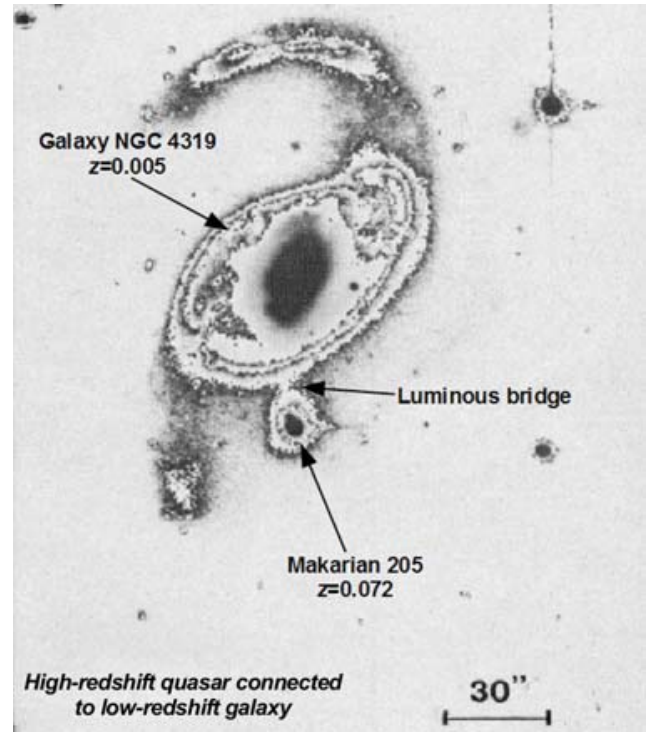

Fig. (1). NGC 4319 and Quasar Makarian 205 apparently connected by a bridge of plasma.

\subsection{Redshift $=$ Recessional Velocity}

Redshift is the name given to a particular interpretation of a feature of observed light which is used in turn to support the theory of the Big Bang and an expanding universe.

An analogy is often drawn between the behavior of light and that of sound, as when the pitch of a siren is heard to increase when approaching and then decrease when receding, hence the term 'Doppler redshift' of light from receding stars. The distance to galaxies, quasars and such has traditionally been defined by Doppler redshift theory. According to this theory, the degree of redshift an objects displays acts as an indication that it is receding from the observer (blueshift = advancing toward the observer) and additionally, that it allows us to calculate the object's distance from us.

The standard we use to determine the redshift and blueshift factors is the light from our own Sun, where light from a distant object is analyzed to determine any amount of deviation from the standard, i.e. if it's redshifted or blueshifted.

Though Edwin Hubble is often credited with advancing the theory of an expanding universe with his work on redshift measurements, he himself was not as sure of the theory as we are often led to understand:

“... it seems likely that red-shifts may not be due to an expanding Universe, and many of the speculations on the structure of the universe may require re-examination" [8].

It was astronomer and former student of Hubble, Halton Arp who investigated and identified two separate components to redshift, intrinsic and motion related [9]. That is to say, that objects have a certain amount of redshift inherent to them regardless of motion. It follows that the redshift/distance assumption is an unreliable guide for calculating the distance of objects in the cosmos.

Arp and his colleagues found that many objects which displayed vastly differing redshift measurements were likely to be in physical association with each other. One example (Fig. 1) showed that galaxy NGC4319 (at 80 million LY

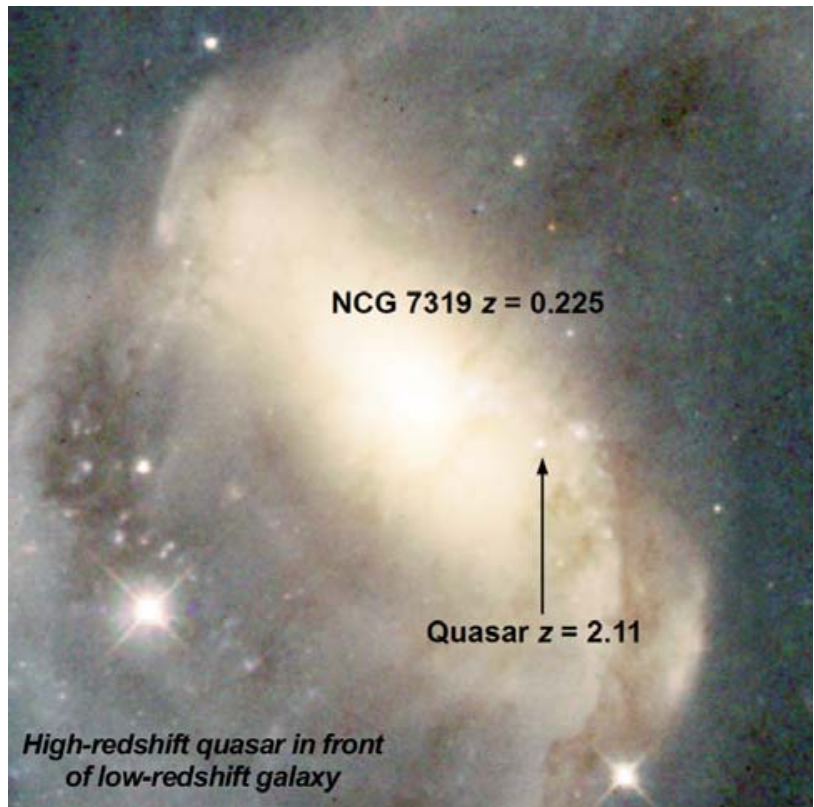

Fig. (2). High-redshift quasar apparently in front of low-redshift galaxy NCG 7319.

distant) and quasar Markarian205 (at 1 billion LY distant) were apparently connected by a bridge of plasma $[10,11]$.

Another example (Fig. 2) shows a highly redshifted quasar which is observed to be in front of low redshift and visually opaque galaxy NGC 7319 [12]. According to conventional wisdom, the quasar is 90 times more distant, than the galaxy it's in front of! The higher the redshift value, the greater the distance according to current theory.

Arp has cataloged many such anomalous findings, yet his observations are largely ignored.

\subsection{Black Holes}

The term 'black hole' was not coined until the 1960's by physicist John Wheeler [13]. It was then used to describe a 'mathematical singularity' said to originate from Karl Schwartzchild's 1915 solution to Einstein's field equations, though a proper mathematical treatment of Schwartzchild's solution renders a singularity impossible [14-17].

Big names including Eddington, Chandrasekhar, Finkelstein and Kerr all drove their fingers into the pie, even though, according to G. t'Hooft, LeMaítre established in 1933 that Eddington's earlier results yielded only a "fictitious singularity" [18]. Though Subrahmanyan Chandrasekhar won the Nobel Prize for physics in 1983 "for his theoretical studies of the physical processes of importance to the structure and evolution of the stars", he had the following to say of black holes:

"The black holes of nature are the most perfect macroscopic objects in the universe: the only elements in their construction are our concepts of space and time" [19].

Nevertheless, mathematicians and theoretical physicists kept juggling equations, bolstered by Steven Hawking's apparent genius at developing thought experiments. The inevitable result being that only 'highly trained specialists' really 'understand' black holes, and the common folk just have to accept their reality. 


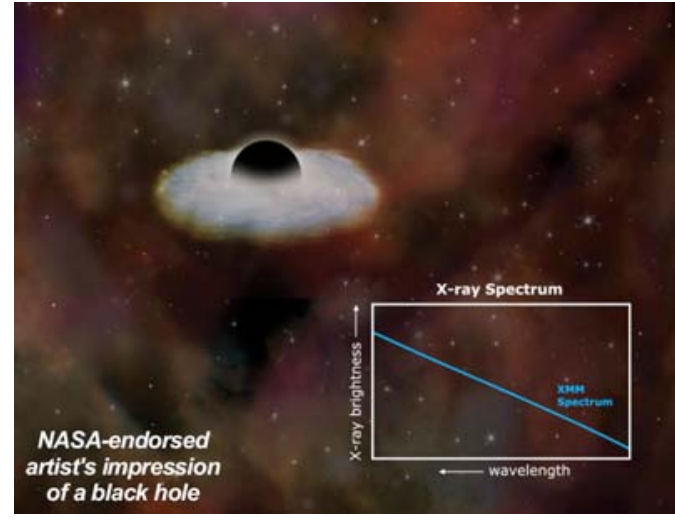

Fig. (3). NASA endorsed artist's impression of a black hole.

Despite many claims to the contrary, black holes have never been found. It has been the choice of astronomers to interpret certain cosmic observations in a way that allows them to believe that black holes are real. They quote the emissions of x-rays, gamma rays and ultraviolet light as evidence of the existence of black holes. This array of phenomena is far better explained through 'synchrotron radiation' the energy type behind most celestial radiation detected here on Earth - and other features of intergalactic electric currents.

Synchrotron radiation is generated when charged particles are accelerated to near light speed along spiral paths in a magnetic field. 'Normal' black holes couldn't produce the required energy to do this, so 'super-massive' black holes were invoked. These were supposed to be created not from millions but from billions of sun masses, an approach which also fitted well with the overall need to explain how galaxies held together if gravity was to be the prime mover of the cosmos.

NASA employs artists to draw pictures of black holes and their surrounds, which usually look nothing like anything we actually observe in space.

The image above (Fig. 3) and many like it would be laughable if not for the seriousness of such being considered an accurate rendition of reality by media and students, and portrayed as such (by implication) by those who create them and endorse their use. To the eye of young and impressionable students, the addition of an x-ray spectrum graph may add credence to an otherwise child-like vision of what a black hole may look like, though without any quantification the graph is meaningless.

\subsection{Dark Matter}

The theory of dark matter was posited in the 1930s by astronomer Fritz Zwicky, in an attempt to address an anomaly within gravity-centric cosmology [20]. Having already bought into the story of gravity being responsible for everything in the heavens, science had to come up with an explanation of the observation that there wasn't enough actual matter in the universe to keep galaxies together, and to explain their rotation curves [21].

To fix this, theorists postulated the existence of dark matter and placed just enough of it (5 times the amount of regular matter!) selectively where needed to make their math work. Dark matter has never been verified experimentally.

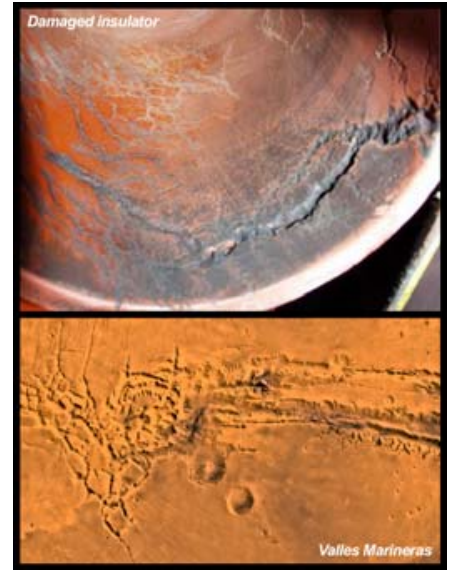

Fig. (4). Top: Insulator damaged by electrical discharge. Bottom: Portion of the Valles Marineras on Mars.

\subsection{Dark Energy}

Putting dark matter in place and relying on redshift became problematic, for it was discovered that other issues had been created, not the least of which was that not only did the universe now appear to be expanding, but also that the rate of expansion was accelerating. A force was needed to overcome the attractive force of gravity to allow for this expansion. Michael Turner, a theoretical cosmologist, coined the term 'dark energy' in 1999 [22] as a name for this 'outward force' (at $72 \%$ of the composition of the universe!) and as a contribution to the idea of 'inflation' (negative gravity!).

The unfortunate result of positing dark matter and dark energy is that together, they account for around $96 \%$ of the composition of the universe. This should have rung alarm bells in the halls of academia.

Adding fuel to the darkening fire, we now have dark flow [23] as a 'mysterious force' acting on galaxy clusters, yet another ad hoc adjustment to the Standard Model. It seems that as long as gravity is the major force available in the cosmos, 'anything goes' including serious consideration of extra dimensions and parallel universes.

\subsection{Craters and Planetary Scars}

Non-volcanic craters are said to be only the result of the high velocity impacts of projectiles on a larger body. The common context is that of small rocky bodies scooting about in space and smashing into larger bodies like planets and asteroids as a result of gravitational forces. Other planetary scarring is said to be due to extreme impact forces on the body in question, or as a result of tectonic movement initiated by internal temperatures or external forces, or volcanic activity, or erosion. It seems strange that we rarely see oblong craters formed by the very probable oblique strikes of flying bodies, and that scarring frequently occurs on bodies that can never have been subject to tectonic, volcanic or erosive forces. An attempt is made to account for the lack of oblong craters by invoking the explosiveness of highvelocity impactors [24]. Whilst this may account for some craters, particularly deep-dished ones, it fails to account for the ubiquitous flat-floored craters, those with central peaks, terraced rims and 'debris fields' aligned tangentially to the point of impact, as observed on many 'rayed' craters [Supplement 1, Fig. 4a]. 


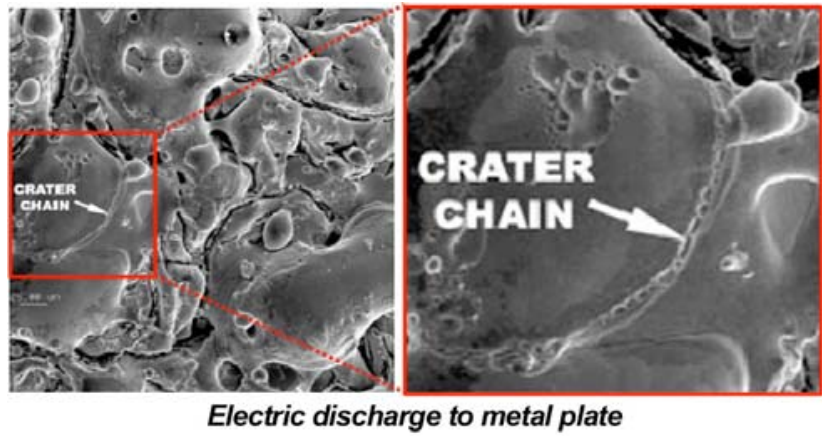

Fig. (5). Uncontrolled electric discharge to metal plate showing clusters of craters, 'rimshot' craters and a crater chain.

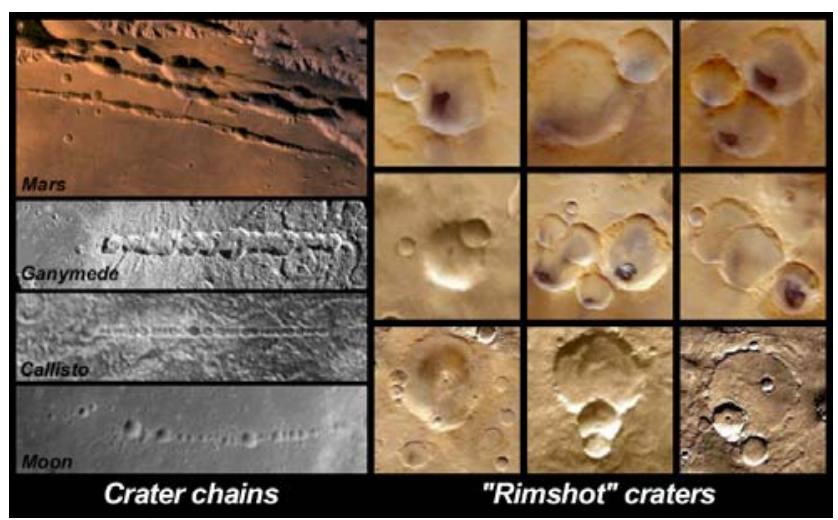

Fig. (6). Montage of crater chains and rimshot craters.

Much of the cratering and scarring observed on planets, comets and asteroids, can have vastly different origins in a plasma universe. The main process involved is tested and proven, and is in practical use here on Earth today - Electric Discharge Machining (EDM). EDM is an industrial process used to produce items of complicated design from metal and other materials. Carefully controlled electrical discharge currents are used to etch, cut and coat all kinds of materials.

Compare the images in Fig. (4). The top is an insulator which has suffered discharge damage, the bottom, a portion of the Valles Marineras on Mars. Present in both images are: crater chains; deep troughs or gouges; shallower trackways; and circular craters with central peaks. These similarities should surely prompt further investigation, at the very least.

Fig. (5) is an example of what can happen when a metal surface is subjected to an uncontrolled electric discharge.

Note the crater chain, and also the group of craters above the second letter " $R$ " which shows a cluster of craters, including small craters perched on the rims of larger craters ('rimshot' craters). These features are ubiquitous both in Earth-application electrical discharges and on planetary bodies. Fig. (6) above, displays crater chains on various solar system bodies, and rimshot craters on Mars.

A close inspection of Mars' Valles Marineras (upper left) reveals that many of the so-called channels which make up this huge scar are in fact, crater chains. NASA has acknowledged that the origin of this massive scar is "unknown" [25]. As with crater chains, 'rimshot' craters and clusters of craters are abundant in numbers which defy impact theories. The occasional incidence of such features would be merely a curiosity, but their sheer number should suffice to force a re-

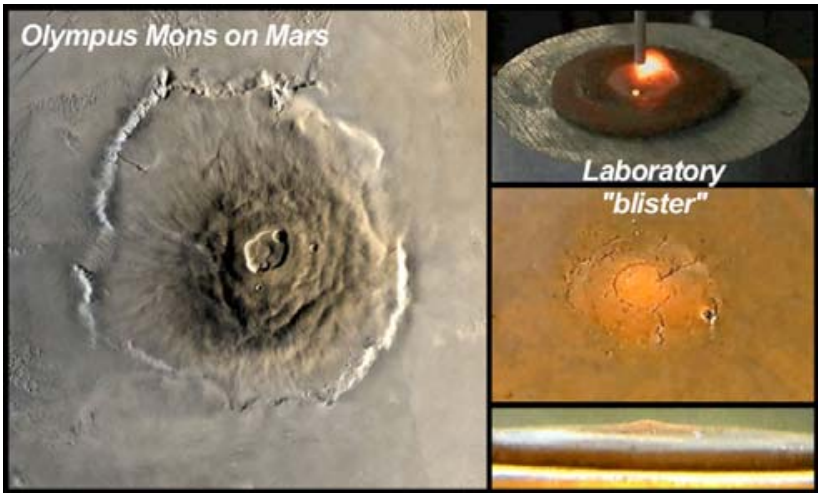

Fig. (7). Left: Olympus Mons on Mars. Right: Electric discharge blister which closely resembles Olympus Mons.

think. Both of these features are common aspects of electrical discharges.

The striations on Phobos, a moon of Mars, appear upon close inspection to be made up almost entirely of crater chains [Supplement 2, Fig. (6a)].

"The origin of these grooves is still debated. It is not known whether they are produced by ejecta thrown up from impacts on Mars, or if they result from the surface regolith, or soil, slipping into internal fissures" [26].

In light of the facts that the grooves or striations completely circumnavigate the body, and that in places they occur at several different orientations, and that on close inspection the majority appear to be crater chains, the above explanation seems rather weak at best. The surface features of Uluru (Ayers Rock) in central Australia are a remarkable match for those of Phobos [Supplement 3, Fig. (6b)].

The higher resolution images of solar system bodies available to us today should have us reconsidering much of planetary geology. With an appropriate level of science funding, plasma experimentalists would be capable of conducting experiments to reproduce many planetary features accurately. As it is, left to their own very limited resources, they are still able to produce accurate analogs for some.

In a small private laboratory in Canberra, HV Engineer Rod Browitt and electrical theorist Wallace Thornhill produced an anode 'blister' on clay, which closely resembles Olympus Mons on Mars, as shown in Fig. (7).

American Plasma physicist C. J. Ransom and Thornhill have also produced craters with spherules and spherules without craters, analogous to those found on Mars using plasma discharges to various compounds including hematite, of which the Martian "blueberries" are thought to be composed [27].

Independent researcher D.Z. Parker has cataloged a collection of simple experiments he has performed in his home [28]. Using such basic materials as disused CRT monitors and dust of varying compositions, he and a colleague have produced remarkably accurate analogs of several planetary features, (Fig. 8) including those mentioned above.

Fig. (8) (left) shows a crater chain on a dust-covered CRT which compares favorably with the crater chains of Ganymede and Callisto in Fig. (6). On the right is a close-up of the dust-covered filter of an ionizing air-purifier. A probe 


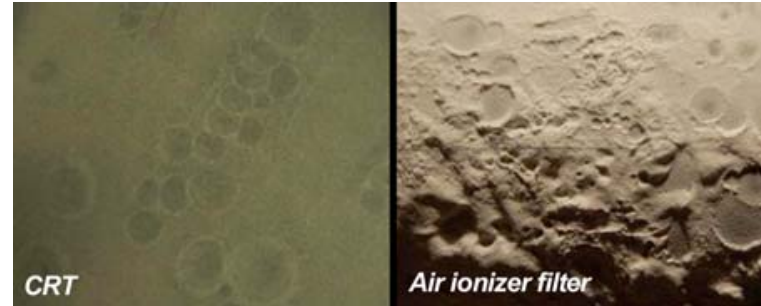

Fig. (8). Left: Crater chains formed on a dusty CRT. Right: Craters on the dust filter of an air ionizer purifier.

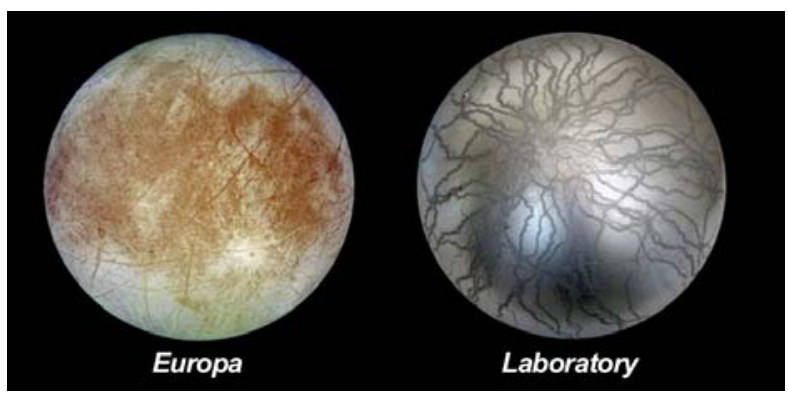

Fig. (9). Left: Europa. Right: Discharge to aluminum coated ball.

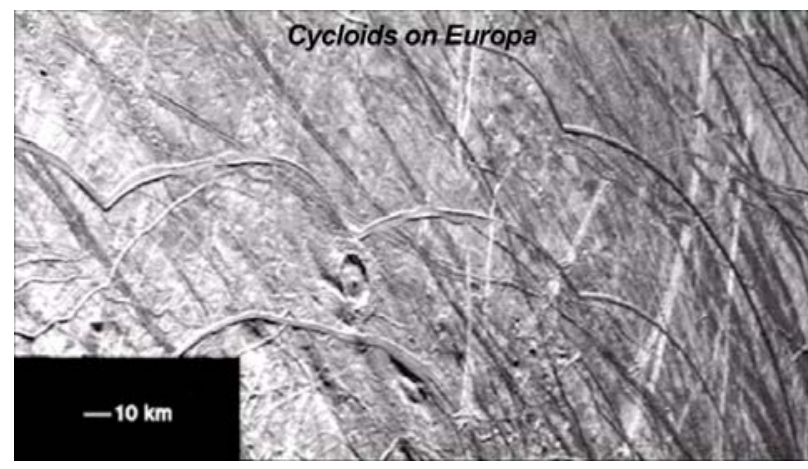

Fig. (10). Cycloids on Europa.

was moved just above the surface and the resulting discharges produced a remarkable array of craters such that it could easily be mistaken for an image of a solar system body. Parker has also produced remarkable analogs for the "spiders" seen on Mars [Supplement 4, Fig. (8a)].

Europa, a moon of Jupiter, has an array of surface features which simply defy orthodox explanations, for which analogies can be identified with reference to discharge activity.

On the left of Fig. (9) is Europa, and on the right is a rubber ball which has been coated in a thin layer of aluminum paint and then subjected to a discharge. The 'smudges' on the ball which resemble those on Europa are not an artifact of photography, but a result of the discharge itself.

A closer image of Europa (Fig. 10) reveals not only the many straight furrows with heaped-up edges which are normally attributed to cracked ice, but also many cycloids, which defy rational geological explanation. Though many theories have been advanced, they all adopt the cracked ice assumption [29-31]. Some of these run for many hundreds and even thousands of kilometers.

Like Europa, Venus too is practically covered in scars which defy rational explanation until one considers electrical action.

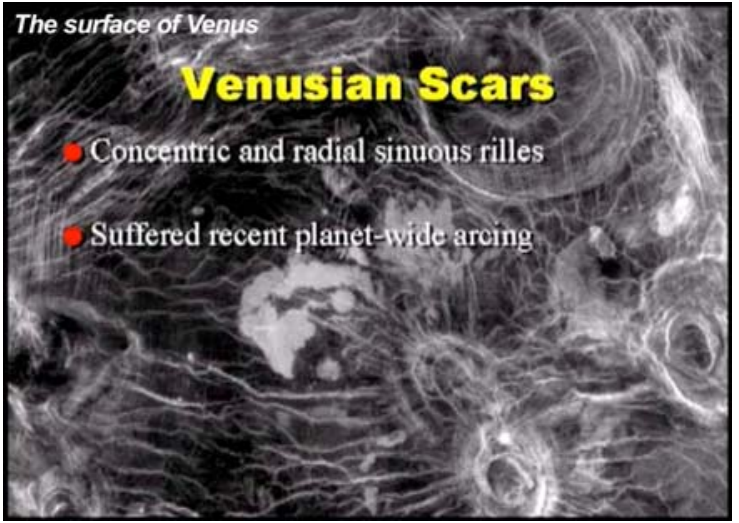

Fig. (11). Concentric and radial rilles on Venus.

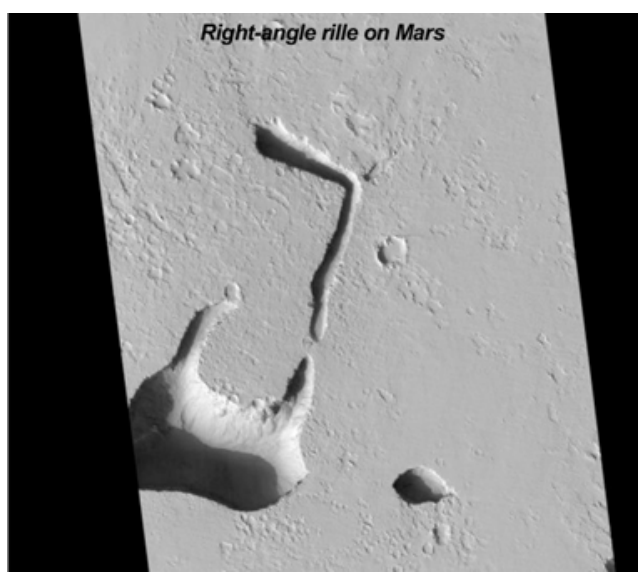

Fig. (12). Right-angle rille on Mars.

Features displaying both concentric and radial rilles (Fig. 11), a rille which is $6,800 \mathrm{~km}$ in length [Supplement 5, Fig. (11a)], huge fulgamites and a straight rille with on-channel craters [Supplement 6, Fig. (11b)], are all difficult to plausibly explain using conventional planetary geology. Thornhill offers electrical explanations which can account for each of these unusual features [32].

One more feature needs further discussion here. 'Sinuous rilles' are a common planetary feature, usually explained by some form of fluid flow, often a lava tube the roof of which has collapsed is invoked. The image above (Fig. 12) of a rille on Mars (of which there are ample examples) defies this or any conventional explanation offered.

Describing this formation as the result of flowing fluids is less than satisfactory. The caption with the original image reads "Channels Emanating from Fissure West of Olympus Mons Aureole" and the image is cataloged under the theme of "Fluvial Processes". Closer views [Supplement 7, Fig. (12a) \& Supplement 8, Fig. (12b)] show that there is no "fissure", there is nowhere at either end of this rille for fluid to have entered or exited, and the right-angle turn where there are no indications of any obstacles to fluid flow is inexplicable.

\subsection{Thermonuclear Theory of Stars}

The Standard Model has it that stars like our Sun are balls of gravitationally compressed hydrogen gas which has become so dense at its core that a nuclear fusion process is initiated. The nuclear reaction is then maintained by the on- 


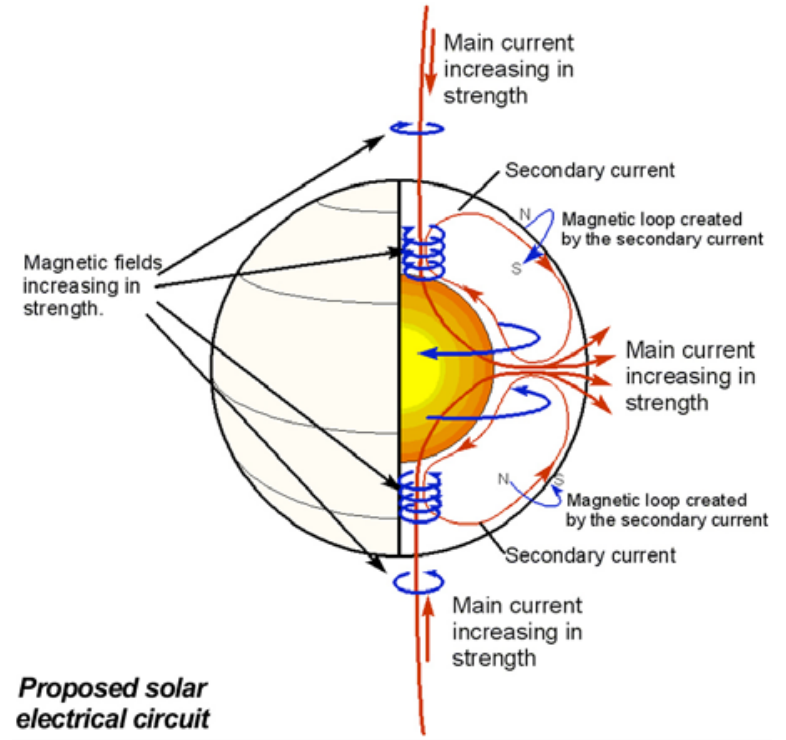

Fig. (13). Proposed electrical circuit of the Sun.

going fusion of hydrogen atoms into helium, a process that will end when the hydrogen runs out. The outward pressure created by the energy from this nuclear fusion process is conveniently balanced by the star's own gravity.

In the fusion model of the Sun, highly energetic photons take millions of years to travel from the core to the surface. In the process they drop in temperature from many millions of degrees to only a few thousand near the photosphere, after which they dramatically increase in temperature again once they leave the Sun. Somehow, the laws of convection are said to account for the strange temperature curve which results.

It was more than 60 years ago that Dr. Charles E. R. Bruce offered a radically different perspective on the Sun:

"[The Sun's] photosphere has the appearance, the temperature and the spectrum of an electric arc; its characteristics are that of an arc, because it is an electric arc, or a large number of parallel arcs" [33].

Years later, engineer Ralph Juergens suggested that the Sun is not an electrically isolated body in space, but the most positively charged object in the solar system; in fact, the center of a radial electric field. This field, he said, lies within a larger galactic field. With this hypothesis, Juergens became the first to make the theoretical leap to an external power source supplying the Sun.

"The phenomena of the photosphere, the phenomena of the chromosphere, the phenomena of the corona, and the known characteristics of the interplanetary medium all fit so nicely into a unifying hypothesis based on energy supplied to the sun from the outside that I cannot resist mentioning it here: I believe that the sun behaves as an anode collecting electric current from its environment, and that the energy it radiates is delivered entirely by way of this postulated electrical discharge" [34].

The Milky Way, just like all galaxies, forms part of an intergalactic electric circuit. The star systems inside it, our own solar system included, are all part of the circuitry. A reasonable analogy would be the systems within a valve amplifier or an analog television set, with vacuum tubes and electric currents doing much of the work. In our solar system the Sun behaves as an anode or positive terminal, and the heliosphere is a huge virtual cathode. Negative ions drift towards the Sun due to charge difference, and heavier positive ions are ejected and accelerated away from the Sun, as the solar wind.

Internal to the Sun (Fig. 13), current flow causes a transformer action, where the varying magnetic fields produced can explain the Sun's magnetic field reversals, sunspot behavior, the differential rotation at various latitudes, and other observed aspects of the Sun's behavior [35, 36].

In the electric model the core of the Sun is relatively cool, the surface is plasma in arc mode, and nuclear reactions take place in the photosphere, which accounts for neutrino production. An externally powered sun will be hotter on the outside than on the inside, which is in agreement with observations, even down to the cooler depths of sunspots, below the photosphere. The Standard Model has it that sunspots are the result of convection and complex magnetic effects, a claim which solar physicists cannot plausibly explain. Only electric currents can produce magnetic fields, so what then creates and sustains them in the gravity model of the Sun if electricity is ignored?

\subsection{Neutron Stars and Pulsars}

A neutron star is said to be the remnant of a supernova explosion, the collapsed core material left behind from the original star. It is said to be crushed so tightly that gravity overcomes the repulsive force between the protons and the electrons so that the atoms collapse resulting in a mass of at least 1.4 times our solar mass compressed into a sphere of around $16 \mathrm{~km}$ diameter, or even less. Pulsars are a special category of spinning neutron star which are said to radiate 'beams of light' or radio pulses, hence their name [37].

Despite the horrendous centrifugal forces involved, neutron stars are said to spin at barely imaginable speeds, some in the order of many tens of thousands of revolutions per minute (rpm). The fastest spinning pulsar to date, J1748$2446 \mathrm{ad}$, has a spin-frequency of $716.358 \mathrm{~Hz}$ [38]. With an estimated diameter of less than $16 \mathrm{~km}$, and spinning at almost 43,000 rpm, it would have a surface speed of about $36,000 \mathrm{~km} / \mathrm{s}$, which is in excess of 129 million kilometers per hour!

The nature of neutron stars and pulsars is very much in question. They have never been observed to the extent that any of this theory can be confirmed, but rather their existence is inferred from observations. Experiments show that neutrons cannot stay together and remain stable, and we know that individually they will decay into a proton and an electron within 15 minutes. The whole notion of these objects seems only to act as a convenient crutch for the work of gravitational theorists.

\subsection{Comets as Dirty Snowballs}

The Standard Model has it that these occasional visitors to our near-Earth space, often referred to as "dirty snowballs", were formed at the icy edges of our solar system, and then locked into a long period orbit around the Sun. As comets approach the Sun, they are said to be heated by solar ra- 


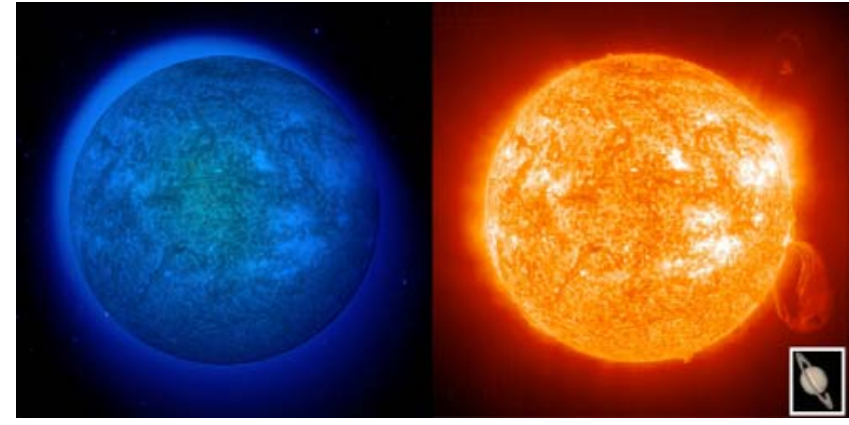

Fig. (14). Coma of comet $17 \mathrm{P} /$ Holmes compared in visual size to the Sun and Saturn.

diation to the point where their ice sublimates. This direct conversion of a solid to a gas produces an envelope of expelled material known as a coma. At the same time, the solar wind of positive ions creates a tail of lighter particles, which always points directly away from the Sun. A second tail can often be seen extending from the coma [39].

Almost every direct observation of comets has produced "surprising" results. Comets don't act at all like dirty snowballs. They frequently display collimated jets originating from all sides including those not facing the Sun, and which do not behave as neutral gases in a vacuum. Their surfaces display sharp relief, not what one would expect from melting ice, and they often display high temperatures and emit x-rays [40].

Despite frequent claims of water being discovered on comets, it is feasible that in some cases the combination of one oxygen atom and one hydrogen atom to form $\mathrm{OH}$ has been misinterpreted as $\mathrm{H}_{2} \mathrm{O}$ simply because water is expected by the Standard Model. $\mathrm{OH}$ is predicted by electrical action [40]. Minerals that can only be formed as the result of extremely high temperature processes have also been found [41], and comets have been known to flare up and disintegrate far away from the Sun.

The currently accepted idea is that their tiny nuclei, often only a few kilometers across can generate a glowing coma of dust that can be hundreds of thousands of kilometers in diameter, and that this is held in place by gravity. A spectacular challenge to this was provided by comet $17 \mathrm{P} /$ Holmes in 2007. In just a matter of days, Holmes grew from a faint magnitude 16.5 to that of 2.8 [42] (over a million times brighter) and was clearly visible from Earth. It soon became the largest object in the solar system, larger in diameter than the Sun. when its coma grew from $28,000 \mathrm{~km}$ to a diameter of 1.4 million $\mathrm{km}$ [43]. The explanations offered for the cause of this event were many and varied, but none gave a satisfactory resolution to the matter.

Fig. (14) shows a comparison of the size of the coma of Comet $17 \mathrm{P} /$ Holmes (1.4 million $\mathrm{km}$ in this image) compared to that of the Sun, and Saturn for good measure. The nucleus of this comet is estimated to be around $3.4 \mathrm{~km}$ across.

Thornhill and Talbott [40] view comets not as dirty snowballs, but as (mostly) rocky bodies with little differentiating them from asteroids apart from their elliptical orbits and evident electrical activity, this concept being based on the earlier work of Ralph Jeurgens.

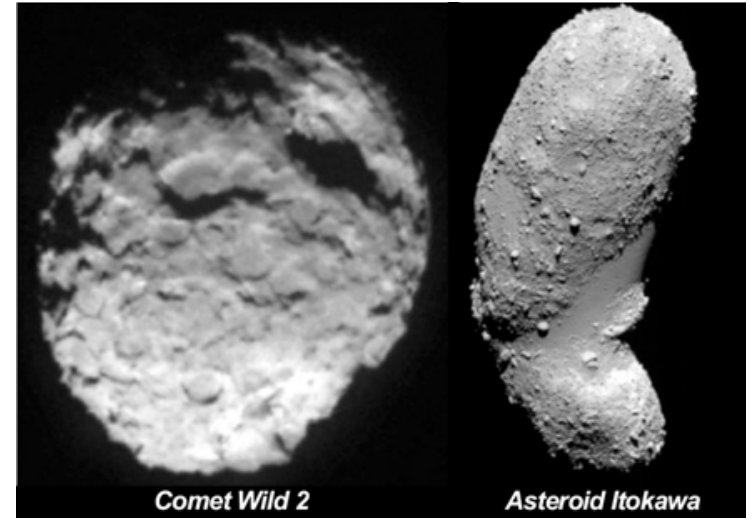

Fig. (15). Comparison of surfaces of a comet and an asteroid.

In the early 1970's, Juergens proposed that comets are negatively charged bodies with respect to the Sun that experience great electrical stresses as they move rapidly into the more positively charged environment near the Sun. Comets spend most of their time in the cold, more negatively charged outer reaches of the solar system, where their charge level is greatly lowered in relation to the highly positively charged Sun that they will eventually come close to [40].

As they approach the Sun, the effect of the rapidly increasing potential difference (voltage between the two bodies) makes their plasma sheath expand and glow, and arcing to the comet nucleus eventually takes place as the comet becomes a 'cold cathode'. Jets of plasma are observed as the comet's charge tries to equalize within the Sun's vast more positive plasma. As the comet's surface is eroded by discharges, sharp features are created, much like the industrial process of EDM as already discussed in the section on craters and planetary scarring. A process known as 'cathode sputtering' takes place which breaks material down to charged particles, fine dust and debris, which is launched away from the comet. Heavier particles follow a different path from the charged and much lighter ions, contributing to the coma and often two tails as comets go around the Sun [40].

Comet nuclei are often blacker than soot. This could be because they have been burnt by electric arcs. They can explode like an over-stressed capacitor, as displayed by comet Shoemaker Levy 9. One of the differences between comets and asteroids in a plasma universe is that comets will have less dust and debris on their surfaces due to the cleaning action of electrical discharge and the expulsion of material into space. This could explain the relatively clean comets and rubble-strewn asteroids frequently seen [40] (Fig. 15).

In 2005 the Deep Impact mission sent an 8201b copper projectile smashing into comet Temple1 at 23,000 mph. It was expected that a small explosion would occur and that this would result in a small but measurable crater being formed, and that the ejected material could be analyzed. The results were anything but what was expected by adherents to the Standard Model.

"The collision sent a huge, bright cloud of debris upward and outward from the comet. Scientists were surprised to learn that the cloud was not composed of water, ice, and dirt. Instead, Deep Impact's instruments indicated that the huge cloud was made up of very fine, powdery material. Due to 
the massive amounts of dust, science team members can only estimate the size [sic] crater's size to be about 325 to 825 feet in diameter" [44].

However, the results of the collision were correctly predicted by Australian physicist Wallace Thornhill when he wrote (along with other things) that there would be a double 'flash' consisting of a powerful electric discharge event prior to a very large impact event which would be more explosive than expected, and that radio communication would be interrupted. He also wrote that more jets were likely to appear, though not necessarily at the site of impact, and that the debris would be largely the same as the surface composition, rather than an abundance of water expected by the Standard Model. Thornhill was vindicated [45], and NASA scientists were left puzzled and surprised.

If predictability is still recognized as a measure of the scientific soundness of a theory, then Thornhill's predictions should lead to serious re-consideration of cometary theory.

\section{THE PLASMA UNIVERSE PERSPECTIVE}

There's not much to be gained from noting problems with the Standard Model without offering something substantial and credible in place of it. Not only does plasma cosmology highlight many problems with the Standard Model, it also offers sound alternatives based on the work of pioneers, which in some cases goes back a century or more.

"It is asserted by Mr. R. A. Proctor, Prof. Osborne Reynolds, and possibly others, that comets owe many of their peculiar phenomena to electric action" [46].

"It seems to be a natural consequence of our points of view to assume that the whole of space is filled with electrons and flying electric ions of all kinds. We have assumed that each stellar system in evolutions throws off electric corpuscles into space. It does not seem unreasonable therefore to think that the greater part of the material masses in the universe is found, not in the solar systems or nebulae, but in "empty" space" [47].

Most astronomers now accept that over $99 \%$ of the visible universe is plasma [48]. Birkeland's position has been vindicated not only with this prediction (though the term 'plasma' had not yet been coined) but also with regard to the field-aligned currents which now bear his name. Even so, astronomers and cosmologists still have a tendency to ignore the role of electric currents in space. Though this situation was perhaps understandable when space was thought of as a 'perfect vacuum', in the light of the work of Birkeland and others after him it is totally unacceptable and needs to be addressed.

Though a detailed treatment of electromagnetism is not within the scope of this paper, a few very basic facts should be stated, as they are very important to the overall theory being discussed.

\subsection{Electricity}

An electric current is the movement of charged particles in a common direction. When a current flows, it generates a magnetic field in a particular direction around the conducting material. When a conducting material moves to cut across a magnetic field, current flow is induced in the conductor. The interaction of a changing current and a changing magnetic field results in the generation of electromagnetic waves. The more efficient and dense the conductor (i.e. the lower its resistivity) the more current it can carry and the stronger the magnetic field that can be created around it.

\subsection{Magnetism}

Magnetism is a force of attraction or repulsion based on the physical orientation of particles within a material. It cannot be maintained without the movement of electrons, ie. an electric current. 'Permanent' magnetism in a material arises from an inherent action within atoms, where the orbits and spin of electrons combine to produce magnetic fields as they orbit their respective nuclei. When atoms within a material become communally aligned through the influence of internal currents or external forces, the material itself (or conductor) takes on a North-South polarization. All materials are influenced by magnetic forces to lesser or greater degrees.

An accurate treatment of magnetism and its relationship to electric currents is offered by Scott [49] in which he explicitly shows that not only can a magnetic field not exist independent of an electric current, but also that the common concepts of "magnetic reconnection", and "field lines" "frozen into" plasma are violations of Maxwell's equations. Boris Somov [50] also gives an explanation of magnetic reconnection which includes consideration of electric currents, something many textbooks ignore.

\subsection{Plasma}

Plasma is a distinct state of matter (usually a gas when referring to space plasmas) in which a significant number of its particles are electrically charged, sufficient for its electrical properties and behavior to be affected.

In an ordinary (electrically neutral) gas individual atoms each hold an equal number of positive and negative charges. When a significant number of atoms in the gas release some or all of their electrons due to the addition of heat or other forms of energy, the gas becomes an 'ionized gas', or plasma. The remaining parts of the atoms which have lost their electrons become positive ions, and the electrons themselves, free to move about, are negative ions.

Plasmas are excellent conductors of current (much better than copper or gold), though NOT perfect conductors. When current flows within plasma, it creates a magnetic field. Plasmas are prodigious producers of electromagnetic radiation [51].

\subsection{Plasma in the Universe}

"The importance of electro-magnetic forces cannot be overstated; even in neutral hydrogen regions $\left(10^{-4}\right.$ parts ionized) the electromagnetic force to gravitational force ratio is still $10^{7 "}$ [48].

The universe is awash with electromagnetic radiation, where the form and frequency of this radiation supplies us with information about what is 'out there'. Highly sensitive optical and radio telescopes, together with new satellite based detectors, look for this deep-space radiation. Where current-carrying plasmas interact, electromagnetic radiation is produced. 

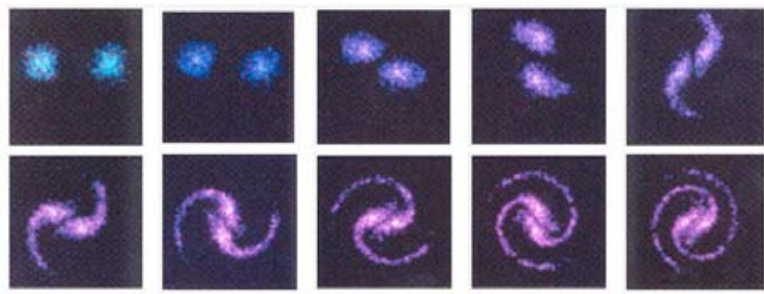

Peratt's PIC galaxy simulation, colored

Fig. (16). PIC galaxy simulation, simplified and colored. No dark matter required.

Where separate plasma regions meet, a structure akin to a cell-wall, known as a Double Layer, is formed which effectively prohibits the two plasmas from meeting directly, and across which almost all of the electric potential (voltage) between the two plasmas will manifest.

Where a neutral plasma interacts with a current-carrying plasma, a current will be induced in the neutral plasma, which will then produce its own magnetic field. These magnetic fields squeeze down on the plasmas they surround, causing a 'pinch' and creating instabilities which add more variables to be considered.

Interacting filaments of current-carrying plasma will be attracted to each other, yet due to the close distance electrical repulsion will not 'touch' and thus they wind around each other creating a natural rotation, and are known as Birkeland Currents.

This constant interaction between various plasmas is why they are inherently difficult, but not impossible, to model mathematically. The continuing feedback between the varying electric currents and the magnetic fields they generate creates a dynamic system which requires re-calculation at every separate time interval and thus it can take extended periods of supercomputer time to model the simplest of interactions.

Nevertheless such modeling has been achieved, and with startling results:

Fig. (16) shows a Particle-In-Cell simulation of spiral galaxy formation performed by Dr. Anthony L. Peratt, arguably one of the world's leading experts in plasma research, at the Los Alamos National Laboratory. The simulation was performed on supercomputers using interacting Birkeland currents and the gravity inherent to the particles [52]. Note that no dark matter was required.

\subsection{Galaxy Structure}

Computer models based on verified plasma science, show that two interacting Birkeland current filaments can reproduce the fine detail and motion of spiral galaxies. These simulations were developed using principles learned in the plasma laboratory. Plasma phenomena are scalable by many orders of magnitude (experimentally at least 14 and hypothetically at least 27 orders of magnitude [53]). Plasma discharge will therefore produce the same basic patterns at laboratory, planetary, stellar, and galactic levels.

Plasma cosmology thus suggests that the continuous flow of Birkeland currents not only creates and holds galaxies together but also drives them like a homopolar motor (the same theory as the revolving disc in household electricity

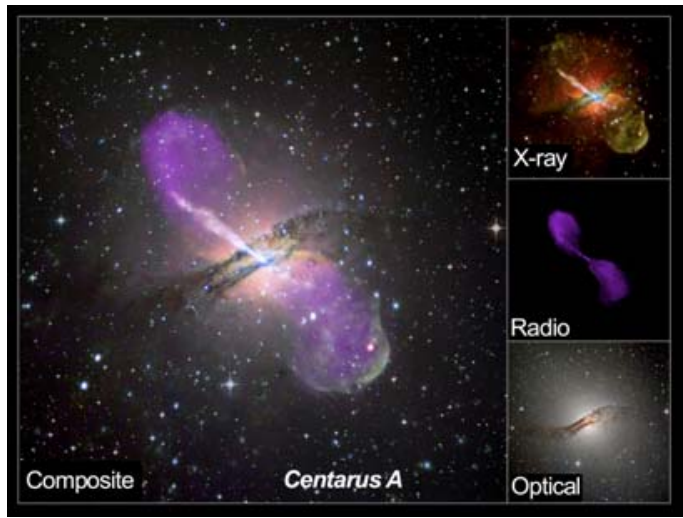

Fig. (17). Centaurus A electromagnetic emissions.

meters). It supplies stars and planets with the charged environment in which they are born, live and die.

In contrast, gravity model computer simulations must assume an initial rotation and rely on selectively placed areas of dark matter in order to force the simulation to duplicate the required structure.

\subsection{Electromagnetic Emissions}

Fig. (17) is a false color composite image of the nearby radio galaxy Centaurus A which displays very high-energy events. Spectacular jets along the axis of the galaxy emitting high-energy synchrotron radiation, two huge radio lobes and an abundance of X-ray sources including ULXs (Ultra Luminous X-ray sources). The right-hand images are X-Ray, Radio and Optical respectively.

The jet's electric current generates its own magnetic field which maintains the helical, pencil-thin beam over vast distances, the upper jet being over 13,000 light years in length. The Chandra release offers the usual "black hole" and "shock waves - akin to sonic booms" to explain the x-ray emissions of the inner parts of the jets, and admits the origin of the more diffuse $\mathrm{x}$-rays in the outer parts of the jets is "not known". The radio lobes are said to be "powered by the central black hole" [54]. In a gravity-only environment there is no plausible mechanism for creating such jets and the powerful magnetic fields and radiation as intense as is seen here.

\subsection{Supernovae and Nebulae}

It is not yet understood why supernovae in faint galaxies should fade faster (relatively) than those in bright galaxies. If supernovae characteristics were related solely to the individual properties of the stars in question, as the Standard Model posits, then the events associated with them should all look similar in magnitude and effect.

Thornhill and Talbott suggest that stars are 'lit' by power from the galaxies themselves [35]. Faint galaxies that don't have as much internal power available as bright galaxies would explain the fading effects observed. Reliance on supernovae brightness to calculate their distance, as is currently done, would therefore be invalidated.

Astronomers believe that supernovae leave in their place either a black hole, a neutron star, a pulsar, or some other gravity related entity. They observe in detail the remnants of supernova events expecting to see the objects and events they predict, but never find them. The gravitational process 


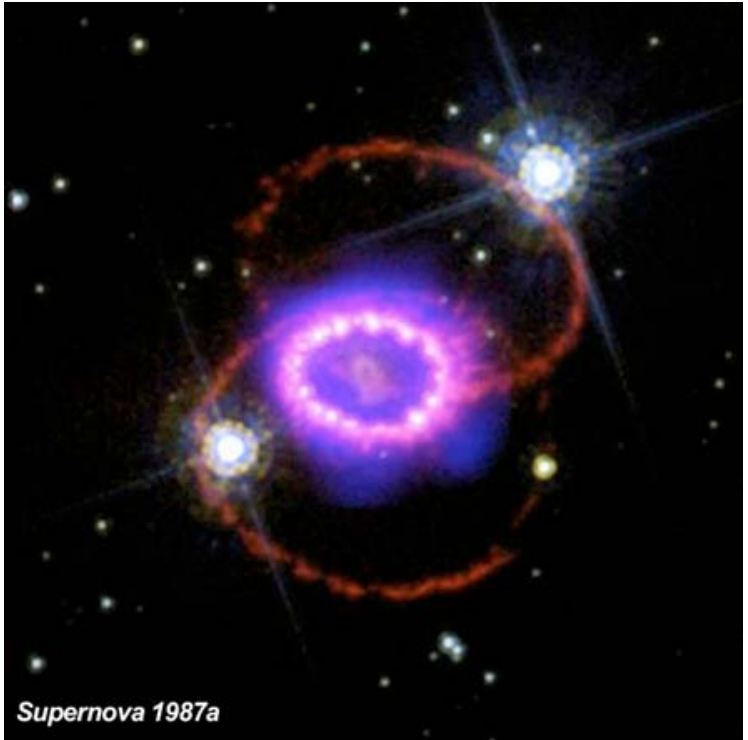

Fig. (18). Supernova 1987a clearly not a spherically symmetrical explosion.

proposed as being responsible for creating these objects out of supernovae events appears much less plausible than Thornhill's explanation based on plasma cosmology [55].

If supernovae are the result of a gravitationally bound object exploding into a vacuum, they should be spherically symmetrical. Most though, are anything but. A striking example is given by figure 18, Supernova 1987a.

The two bright star-like objects are in the background and are not part of the supernova. A very plausable explanation for sn1987a lies in the morphology of a z-pinch in a current-carrying plasma. That gravitation and shockwaves could result in what we see here requires speculation on a grand scale. Yet if we allow for currents to be involved, suddenly it can start to make sense.

The central ring [Supplement 9, Fig. (18a)] is prolific in $\mathrm{x}$-rays which the Standard Model has a great deal of trouble explaining. The description offered in the Chandra album is convoluted and almost desperate in this author's opinion. First a "slow wind" set up a cloud around the original star, which was followed by a "fast wind" which carved a cavity in the debris left by the slow wind. The supernova event then occurred and "sent a shockwave rumbling through the cavity". This shockwave encountered the debris left earlier and produced the x-radiation we see [56]. Nature doesn't do things the hard way. If we see x-rays, they are most likely to be caused electrically, the same way a dentist produces them.

Whilst the Chandra description accounts (weakly) for the central ring, it offers absolutely nothing to account for the two outer rings, as though they do not exist. When viewed as a whole, a z-pinch particle beam simulation reproduces what we see in Supernova 1987a [35] without the need to invoke a ring of debris which has no business being there in the first place [Supplement 10, Fig. (18b)].

The bipolar morphology exhibited by sn1987a is not unusual. Many nebulae also exhibit similar morphology (such as the Red Square nebula [Supplement 11, Fig. (18c)]), and Thornhill argues convincingly for the same z-pinch phenomenon in explanation of these.

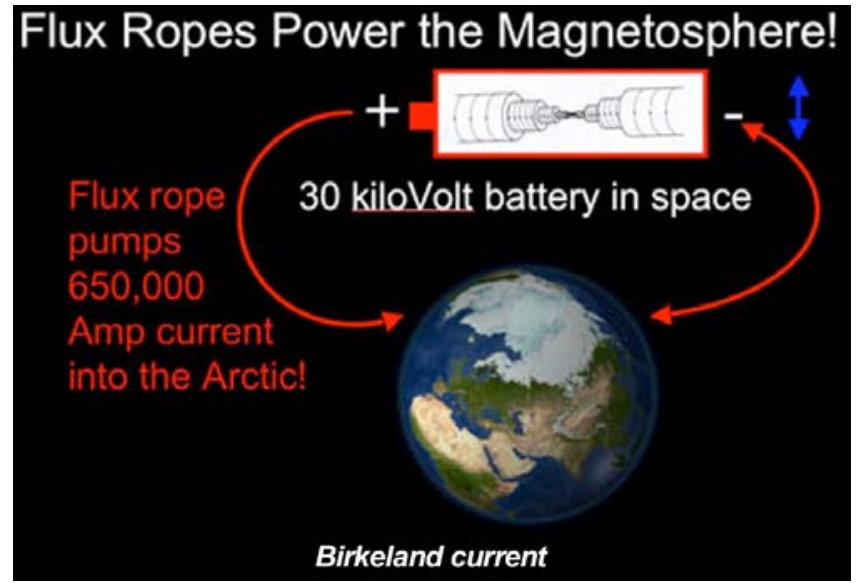

Fig. (19). "Flux Ropes Power the Magnetosphere!: THEMIS discovered a flux rope pumping a 650,000 Amp current into the Arctic" [57].

\subsection{Earth, Lightning and Weather}

If we live in a Plasma Universe, then surely our own Earth would be affected by this, and surely, it is. There is much evidence now which connects the Earth's surface to space, electrically. Lightning is part of just such a circuit. Recent confirmation of sprites, elves and gnomes in the upper atmosphere lends further support. Finally, it was recently announced by NASA that "magnetic twisted ropes" connect the Earth to the Sun (Fig. 19).

We need no more evidence to see that our Earth is part of a much broader electric circuit, enabled by the actions of plasma throughout space, though most science media still insist that the magnetism we observe is 'mysterious' rather than accepting the electrical nature of it. Remember, we cannot have magnetism without an electric current to generate and maintain it, as NASA now appears to recognize at least in this instance.

Lightning is frequently seen in and around volcanic plumes. 'Earthquake lights' are becoming a commonly reported phenomenon, often seen days before the quake itself. Weather forecasting is mainly based on temperature and pressure differences, with no account of electric charges being taken (apart from thunderstorms). Considering our often unreliable forecasting services, what might this suggest about the current science of weather and atmospheric phenomena?

Presently, theory suggests that it is solar heating of the surface of the Earth (both water and land) which creates areas of differing pressure, the highs and lows seeking equilibrium driving our winds and storms. Conveniently, where planets are too far from the Sun, other causes are invoked for essentially the same weather patterns. The wind on Saturn can reach over $1000 \mathrm{mph}$ at the equator, yet Saturn is around 9.5 times more distant from the Sun than Earth is. And as a gas giant, there should be no surface to heat. So what causes its wild weather?

The hexagonal 'storm' on Saturn's north pole shown in Fig. (20) is also very difficult to explain under any theory, but at least we do observe hexagonal morphology in relation to plasma discharges. The possibility that this unusual feature has an electrical cause requires consideration. 


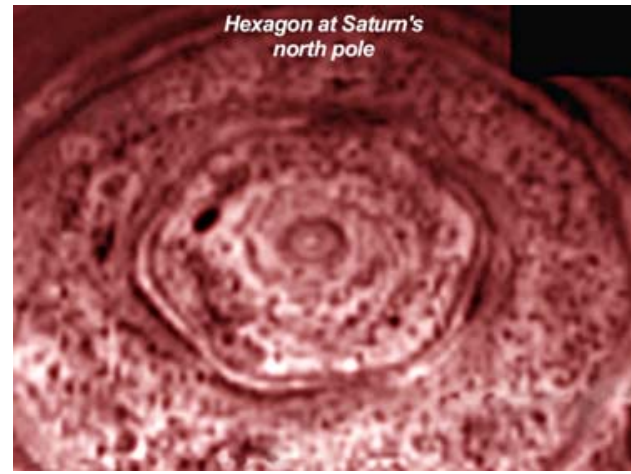

Fig. (20). The hexagon at Saturn's north pole, for which there is no currently accepted explanation.

On Earth we have auroras, lightning, sprites, elves, tornadoes, hurricanes, waterspouts, dust devils and geomagnetic storms. On other bodies in our Solar System we have different phenomena, like giant dust devils on Mars and features which exhibit the appearance of plasma discharges (conventionally described as volcanic plumes) on Io. What influences the weather systems that produce these features?

\section{CONCLUSIONS}

Whilst this paper is far from exhaustive, it hopefully has given readers some food for thought about the state of astrophysics and cosmology today, and indeed some of the other sciences which the Plasma Universe theory touches upon.

Modern cosmology rests upon gravity and, to a lesser degree, 'mysterious' magnetism to organize everything we observe in the universe. It all started, we are told, with a Big Bang where everything which exists today came into being. The universe is, however, largely inhomogeneous throughout. The fact that we can see clumps of physical objects amid vast empty spaces seems to contradict Big Bang theory. If a gas enters a vacuum, it will spread out violently in order to support equalization of pressure and density. How then, realistically, can galactic scale filaments of 'gas' be formed and held together by the weakest force known to physics?

The jets that emanate from quasars and galaxies reach out for hundreds and thousands of light years. How can this be if their so-called gaseous make-up desperately wants to disperse itself within the void? NASA has said it believes this phenomenon can be explained by "mysterious" magnetized gas swirling around massive Black Holes at the centers of these structures. There was a time when nothing could escape the clutches of a Black Hole. Now it seems they can do most anything.

It should be remembered that gravity is a force of attraction only, and that fact itself is fatally limiting when it comes to current explanations of our universe. The electric force however, is two-way - attractive and repulsive - and 39 orders of magnitude more powerful than gravity. This fact which answers many of the puzzles of cosmology still remains largely ignored because most astronomers continue to maintain that separately charged bodies cannot exist in the vast expanses of the cosmos.

The simple fact remains that, considering plasma in space, if only 1 in 10,000 particles is a charged particle, then the resulting force produced will still overwhelm gravity.
Astronomers often refer to plasma as a 'hot gas'. What they don't say is that it conducts electricity, is influenced by magnetic fields, and has properties not normally associated with a gas. Plasma in space is almost electrically neutral, but as differing plasma regions on huge scales move with respect to each other, electric currents are generated within them. The resulting Birkeland currents formed are as a result of the charged particles moving in alignment with the local magnetic field, this being the most efficient path for electric currents to flow in plasma. These Birkeland currents act as cosmic power transmission lines in deep space.

Astronomers observe what they refer to as "surprising" $\mathrm{X}$-ray and radio emissions in space. Their gravitational models require exotic, undetectable and inconsistent theoretical inventions to explain these observations. Working from the well established field of plasma physics, plasma cosmologists are able to explain these observations using known and well proven science. More than that, many of these phenomena can and have been reproduced in the laboratory in a manner that exemplifies the scientific method.

Electric currents across cosmic distances have the power to shape cosmic structure, spin galaxies, illuminate planetary nebulae, energize the stars, light our sun, generate planetary auroras, and create spectacular comets. It is becoming apparent to some scientists that it is the powerful electric force which dominates on the larger scale in the universe. Compared to the electric force, gravity plays only a minor role. The ubiquitous $\mathrm{x}$-rays, gamma rays and extreme ultraviolet radiation detected in celestial gas clouds and coming from galactic jets are not mysteries, but effects that are expected and predicted by the model, based on experiments in plasma laboratories.

It is this author's contention that much of modern science has become homo-centric. We are at the center of everything. Anything we can imagine or dream up is fair game. We can answer all the questions. We no longer require evidence, only a belief that anything is possible.

That black holes, wormholes and parallel universes have been allowed to flourish within the pages of peer-reviewed scientific publications is a sad indictment indeed. It is not suggested that these things are necessarily impossible, just that we should look to nature and the forces we do know before inventing exotic unseen, non-falsifiable and unintuitive entities in a vain effort to appear to be answering the mysteries of the cosmos.

One of the most common questions asked of plasma cosmology advocates is "But where does all this electricity come from? Until you identify a source for it, I won't even consider the theory." And this usually from those who are happy to accept that once upon a time, nothing went BANG and now we have a universe! A valid counter-question would be "Where does all the spin come from?" Though spin is a natural property of Birkeland currents in a plasma universe, it must be an assumed initial condition for it to exist within the big bang paradigm.

The most refreshing aspect of plasma theorists I have observed is that they don't claim to have all the answers. There is much we don't know, and there are some things we are just never likely to know. 


\section{ACKNOWLEDGMENTS}

The author wishes to thank Thomas Findlay for providing initial inspiration for this paper, and Alex Jarvie for assisting with reference materials. Also thanks are due to a number of anonymous colleagues who assisted with thoughtful discussion, corrections and advice. This work was not funded in any way by any body and is entirely the product of the unaffiliated and independent author.

\section{IMAGE CREDITS}

All images in this work are incorporated under the "fair use" provisions of the appropriate copyright legislation.

Fig. (1). NGC 4319 and Quasar Makarian 205 apparently connected by a bridge of plasma. Adapted from:

Sulentic, J. and Arp, H. (1987) The galaxy-quasar connection - NGC 4319 and Markarian 205. I - Direct imagery. II - Spectroscopy, Astrophysical Journal, Part 1 (ISSN 0004637X), vol. 319, Aug. 15, 1987, p. 687-708.

http://adsabs.harvard.edu/abs/1987ApJ...319..687S

Image inverted and annotations added.

Fig. (2). High-redshift quasar apparently in front of lowredshift galaxy NCG 7319. Adapted from:

NASA, J. English (U. Manitoba), S. Hunsberger (PSU), Z. Levay (STScI), S. Gallagher, and J. Charlton (PSU).

http://hubblesite.org/newscenter/archive/releases/2001/22 /image/a/

Cropped and anotations added.

Fig. (3). NASA endorsed artist's impression of a black hole. Adapted from:

Spectrum: ESA/XMM-Newton/S. Komossa et al. Illustration: NASA/CXC/M. Weiss.

http://chandra.harvard.edu/photo/2004/rxj1242/more.html

"This graphic shows the XMM-Newton spectrum, or Xray energy signature, of RX J1242-11 alongside an artist's illustration of the event that was observed in this galaxy. The illustration shows a disk of gas being heated so that it glows in X-rays before being swallowed by the black hole. The gas in the disk is the debris from a star torn apart by tidal forces about 10 years earlier."

Fig. (4). Top: Insulator damaged by electrical discharge. Bottom: Portion of the Valles Marineras on Mars. Adapted from:

Insulator image: EA Technology. Used with permission.

Valles Marineras: NASA/JPL/USGS.

http://photojournal.jpl.nasa.gov/catalog/PIA00422

Fig. (5). Uncontrolled electric discharge to metal plate showing clusters of craters, 'rimshot' craters and a crater chain. Adapted from:

Thornhill, W. Used with permission.

Fig. (6). Montage of crater chains and rimshot craters. Adapted from:

Crater chain montage:

\section{Mars: NASA/JPL/USGS.}

http://photojournal.jpl.nasa.gov/catalog/PIA00422

Ganymede: NASA/JPL/Brown University.

http://photojournal.jpl.nasa.gov/catalog/pia01610

Callisto: Paul Schenk/Lunar \& Planetary Institute.

http://neo.jpl.nasa.gov/images/callisto.html

Moon: NASA/GSFC/Arizona State University.

http://www.nasa.gov/mission_pages/LRO/multimedia/lro images/lroc-20100330-orientale.html

Rimshot montage:

Google Earth (Mars) Credit: ESA/DLR/FU Berlin (G Neukum)

\section{Image: NASA/USGS.}

Fig. (7). Left: Olympus Mons on Mars. Right: Electric discharge blister which closely resembles Olympus Mons. Adapted from:

Olympus Mons:

Image Processing by Jody Swann/Tammy Becker/Alfred McEwen, using the PICS (Planetary Image Cartography System) image processing system developed at the U.S. Geological Survey in Flagstaff, Arizona.

http://nssdc.gsfc.nasa.gov/photo_gallery/photogallerymars.html\#features

Laboratory images: Browitt, R. and Thornhill, W. Planetary Electrical Scarring video, Mikamar Publishing, 2000.

Fig. (8). Left: Crater chains formed on a dusty CRT. Right: Craters on the dust filter of an air ionizer purifier. Adapted from:

Credits: Left - DZ Parker; Right - James St. Pe.

http://www.electric-spark-scars.com

Fig. (9). Left: Europa. Right: Discharge to aluminum coated ball. Adapted from:

Left: NASA/JPL; Right: C. J. Ransom/Vemasat Laboratories.

http://thunderbolts.info/tpod/2010/arch10/100625smallsc ale.htm

Fig. (10). Cycloids on Europa. Adapted from:

Thornhill, W. Planetary Electrical Scarring video, Mikamar Publishing, 2000. from:

Fig. (11). Concentric and radial rilles on Venus. Adapted

Thornhill, W. Planetary Electrical Scarring video, Mikamar Publishing, 2000.

Fig. (12). Right-angle rille on Mars. Adapted from:

NASA/JPL/University of Arizona.

http://hirise.lpl.arizona.edu/PSP_003292_2025

Fig. (13). Proposed electrical circuit of the Sun. Adapted from: 

ing.

Scott, D. E. (2006) The Electric Sky, Mikamar Publish-

Fig. (14). Coma of comet 17P/Holmes compared in visual size to the Sun and Saturn. Adapted from:

Left: CFHT/Rachel Stevenson, David Jewitt (UCLA) and Pedro Lacerda (Queen's Univ. Belfast);

Right: ESA/NASA/SOHO/Voyager.

http://www2.ess.ucla.edu/ jewitt/holmes.html

Fig. (15). Comparison of surfaces of a comet and an asteroid. Adapted from:

Comet Wild 2: NASA/JPL.

http://stardust.jpl.nasa.gov/photo/wild2.html

Asteroid Itokawa: ISAS, JAXA.

http://www.astronet.ru/db/varstars/msg/1210651

Fig. (16). PIC galaxy simulation, simplified and colored. No dark matter required. Adapted from:

LANL/Peratt AL. Retrieved from: Wikimedia commons.

http://commons.wikimedia.org/wiki/File:Peratt's_galaxy_ formation_simulation.jpg

Fig. (17). Centaurus A electromagnetic emissions. Adapted from:

X-ray: NASA/CXC/CfA/R.Kraft et al.;

Radio: NSF/VLA/Univ.Hertfordshire/M.Hardcastle;

Optical: ESO/WFI/M.Rejkuba et al.

http://chandra.harvard.edu/photo/2008/cena/

Fig. (18). Supernova 1987a clearly not a spherically symmetrical explosion. Adapted from:

X-ray: NASA/CXC/PSU/S.Park \& D.Burrows.;

Optical: NASA/STScI/CfA/P.Challis.

http://chandra.harvard.edu/photo/2005/sn87a/

Fig. (19). An obvious electrical connection between Earth and space. Adapted from:

NASA/GSFC.

http://www.nasa.gov/mission_pages/themis/auroras/north ern_lights_multi.html (image \#10)

Fig. (20). The hexagon at Saturn's north pole, for which there is no currently accepted explanation. Adapted from:

NASA/JPL/University of Arizona.

http://photojournal.jpl.nasa.gov/catalog/PIA09188

\section{ACKNOWLEDGMENT}

The author wishes to thank Thomas Findlay for providing initial inspiration for this paper, and Alex Jarvie for assisting with reference materials. Also thanks are due to a number of anonymous colleagues who assisted with thoughtful discussion, corrections and advice. Special acknowledgment is due to Dr. Malcolm Simons for his encouragement and support without which this paper would not have seen the light of day. This work was not funded in any way by any body and is entirely the product of the unaffiliated and independent author.

\section{CONFLICT OF INTEREST}

None Declared.

\section{SUPPLEMENTARY MATERIAL}

Supportive/Supplementary material as available on the publisher web site along with the published article.

\section{REFERENCES}

[1] Hoyle F. Home is where the wind blows. California: University Science Books 1994.

[2] Dicke RH, Peebles PJE, Roll PG, Wilkinson DT. Cosmic blackbody radiation. Astrophys J 1965; 142: 414-9.

[3] Penzias AA, Wilson RW. A measurement of excess antenna temperature at $4080 \mathrm{Mc} / \mathrm{s}$. Astrophys J 1965; 142: 419-21.

[4] Dunning-Davies J. Exploding a myth: 'Conventional Wisdom' or scientific truth? Chichester: Horwood 2007.

[5] Lieu R, Mittaz JPD, Zhang S-N. The sunyaev-zel'dovich effect in a sample of 31 clusters: a comparison between the $\mathrm{x}$-ray predicted and wmap observed cosmic microwave background temperature decrement. Astrophys J 2006; 648: 176-99.

[6] Lerner EJ. Radio Absorption by the intergalactic medium. Astrophys J 1990; 361: 63-8.

[7] Verschuur GL. High galactic latitude interstellar neutral hydrogen structure and associated (wmap) high-frequency continuum emission. Astrophys J 2007; 671(1): 447-57.

[8] Hubble E. The 200-inch telescope and some problems it may solve. Publ Astron Soc Pac 1947; 59(349): 153-67.

[9] Arp H. Seeing red: redshifts, cosmology and academic science. Montreal: Apeirion C. Roy Keys Inc 1998.

[10] Arp, HA Connection Between the Spiral Galaxy NGC 4319 and the Quasi-Stellar Object Markarian 205. Astrophys Lett 1971; 9: 1.

[11] Sulentic J, Arp H. The galaxy-quasar connection - NGC 4319 and Markarian 205 I - Direct imagery, II - Spectroscopy. Astrophys J 1987; 319: 687-708

[12] Pasquale G. The discovery of a high-redshift x-ray emitting qso very close to the nucleus of NGC 7319. Astrophys J 2005; 620: 8894.

[13] Wheeler JA, Ford KW. Geons, black holes, and quantum foam: a life in physics. New York: W. W. Norton \& Company 1998; p. 296.

[14] Abrams LS. Black holes: the legacy of Hilbert's error. Can J Phys 1989; 67: 919 .

[15] Crothers SJ. On the general solution to Einstein's vacuum field and its implications for relativistic degeneracy. Prog Phys 2005; 1: 6873.

[16] Crothers SJ. On the Ramifications of the Schwarzchild Space-Time Metric. Prog Phys 2005; 1: 74-80.

[17] Rabounski D. On the current situation concerning the black hole problem. Prog. Phys 2008; 1: 101-3.

[18] 't Hooft G. Introduction to the theory of black holes. Lectures presented at Utrecht University 2009. Available from: http://www.phys.uu.nl/ thooft/lectures/blackholes/BH_lecturenotes .pdf [Accessed: 2011/03/21].

[19] Chandrasekhar S. The Mathematical Theory of Black Holes. New York: Oxford University Press 1998

[20] Zwicky F. Die Rotverschiebung von extragalaktischen Nebeln. Helvetica Phys Acta 1933; 6: 110-27.

[21] Zwicky F. On the Masses of Nebulae and of Clusters of Nebulae. Astrophys J 1937; 86: 217.

[22] Turner MS. Dark matter and dark energy in the universe. The third stromlo symposium: the galactic halo. Gibson BK, Axelrod TS and Putman ME, Eds. Astron Soc Pac Conf Series 1999; 666.

[23] Kashlinsky A, Atrio-Barandela F, Kocevski D, Ebeling H. A measurement of large-scale peculiar velocities of clusters of galaxies: results and cosmological implications. Astrophys J 2008; 686(2): L49-L52.

[24] Lyzenga GA. Why are impact craters always round? Most incoming objects must strike at some angle from vertical, so why don't the majority of impact sites have elongated, teardrop shapes? Sci- 
entific American Ask the Experts October 21, 1999. Available from: http://www.scientificamerican.com/article.cfm?id=why-areimpact-craters-al Accessed 2011/03/21.

[25] Valles marineris: the grand canyon of mars. NASA Image of the Day Gallery March 23, 2008. Available from: http://www.nasa.gov/multimedia/imagegallery/image_feature_83.html Accessed 2011/03/21

[26] ESA Mars Express, News July 30, 2008. Available from: http://www.esa.int/SPECIALS/Mars_Express/SEM5H48N9JF_0.ht $\mathrm{ml}$ Accessed 2011/03/21.

[27] Ransom CJ, Thornhill W. Plasma-generated craters and spherules. IEEE Trans Plasma Sci 2007; 35(4). DOI 10.1109/TPS.2006.888590 Available from: http://ieeexplore.ie-ee. org/xpl/freeabs_all.jsp?arnumber $=4287076$

[28] Available from: http://members.cybertrails.com/ zrwoaz/ [Accessed 2011/03/22].

[29] Groenleer JM, Kattenhorn SA. Cycloid crack sequences of Europa: Relationship to stress history and constraints on growth mechanics based on cusp angles. Icarus 2007; 193(1): 158-81.

[30] Hurford TA, Sarid AR, Greenberg R, Bills BG. The influence of obliquity on europan cycloid formation. Icarus 2009; 202(1): 197215.

[31] Rhoden A, Hurford TA, Militzer B, Huff E, Manga M, Richards MA. The case for europa's finite obliquity from cycloids and strike-slip faults. American Geophysical Union, Fall Meeting 2009; abstract \#P51E-1165 Available from: http://adsabs.harvard.edu/abs/2009AGUFM.P51E1165R [Accessed 2011/03/22].

[32] Thornhill W. Planetary Electrical Scarring DVD. Oregon: Mikamar Publishing 2000.

[33] Bruce CER. A new approach in astrophysics and cosmogony. London and Woking: Unwin Brothers Limited 1944. Available from: http://www.catastrophism.com/texts/bruce/astro.htm [Accessed 2011/03/22].

[34] Juergens R. Reconciling celestial mechanics and velikovskian catastrophism. Pensee 1972; 2(3) Available from: http://www.catastrophism.com/cdrom/pubs/journals/pensee/ivr02/i ndex.htm Can be read online at: http://saturniancosmology.or-g/ juergens.htm [Accessed 2011/03/22].

[35] Thornhill W, Talbott D. The Electric Universe. Oregon: Mikamar Publishing 2007.

[36] Scott D. The electric sky. Oregon: Mikamar Publishing 2006

[37] NASA/GSFC. Neutron stars, pulsars, millisecond pulsars, and gravitational radiation: a primer. Available from: http://www.nasa.gov/centers/goddard/news/gsfc/spacesci/pictures/2003/0702puls arspeed/0702ssu_primer.html [Accessed 2011/02/24].

[38] Hessels JWT, Ransom SM, Stairs IH, Freire PCC, Kaspi VM, Camilo F. A Radio Pulsar Spinning at 716 Hz. Science 2006; 311(5769): 1901-4.

[39] NASA solar system exploration, planets, comets. Available from: http://solarsystem.nasa.gov/planets/profile.cfm?Object=Comets\&D isplay=OverviewLong [Accessed 2011/03/24].
[40] Thornhill W, Talbott D. The Electric comet. Poster presentation at the IEEE 33rd International Conference On Plasma Science (ICOPS) 2006. Available from: http://www.thunderbolts.info/pdf/ElectricComet.pdf [Accessed 2011/03/24].

[41] NASA/JPL Stardust findings suggest comets more complex than thought. Dec 14, 2006. Available from: http://stardust.jpl.na-sa.gov /news/news 110.html [Accessed 2011/03/24].

[42] Harvard-smithsonian center for astrophysics photometry of comet 17P/Holmes. Available from: http://www.cfa.harvard.e-du/ icq/17P_ICQ.html [Accessed 2011/03/24].

[43] Stevenson R, Jewitt D. Comet holmes bigger than the Sun. UCLA 2009. Available from: http://www2.ess.ucla.edu/ jewitt/holmes.html [Accessed 2011/03/24].

[44] NASA Solar System Exploration, Missions, Deep Impact-EPOXI. Available from: http://solarsystem.nasa.gov/missions/profile.c$\mathrm{fm}$ ?Sort $=$ Nation $\&$ MCode $=$ DeepImpact $\&$ Nation $=U S A \&$ Display $=\mathrm{R}$ eadMore [Accessed 2011/03/24].

[45] The Thunderbolts project: predictions confirmed, comets: deep impact. Available from: http://www.thunderbolts.info/predictions.htm\#cdi [Accessed 2011/03/24].

[46] Jevons WS. Nature 1871; December 28.

[47] Birkeland K. The Norwegian aurora polaris expedition 1902-1903. Christiana: H. Aschehoug \& Co 1908.

[48] Peratt AL. Advances in numerical modeling of astrophysical and space plasmas. Astrophys Space Sci 1997; 242(1-2): 93-163.

[49] Scott D. Real properties of electromagnetic fields and plasma in the cosmos. IEEE Trans Plasma Sci 2007; 35(4): 822-7.

[50] Somov B. Fundamentals of cosmic electrodynamics.UK: Kluwer Academic Publishers 1994; p. 191.

[51] Peratt AL. Physics of the plasma universe. New York: SpringerVerlag 1992.

[52] Peratt AL. Evolution of the Plasma Universe: II. The formation of systems of galaxies. IEEE Trans Plasma Sci 1986; 14(6). Available from: http://public.lanl.gov/alp/plasma/downloadsCosmo/Per-att86 TPS-II.pdf [Accessed 2011/03/24].

[53] Peratt AL. Characteristics for the occurrence of a high-current, zpinch aurora as recorded in antiquity. IEEE Trans Plasma Sci 2003; 31(6), 1192-1214.

[54] Harvard-Smithsonian Center for Astrophysics, Chandra X-ray Observatory. Centaurus A: Black Hole Outflows From Centaurus A. Available from: http://chandra.harvard.edu/photo/2009/cena/ Accessed 2011/03/24.

[55] Thornhill W. Z-Pinch Morphology of Supernova 1987a and Electric Stars. IEEE Trans Plasma Sci 2007; 35(4): 832-44.

[56] Harvard-Smithsonian Center for Astrophysics, Chandra X-ray Observatory. Supernova 1987A: Fast Forward to the Past. Available from: http://chandra.harvard.edu/photo/2005/sn87a/ [Accessed 2011/03/24]

[57] NASA Themis: understanding space weather. Available from: http://www.nasa.gov/mission_pages/themis/auroras/northern_lights _multi.html [Accessed 2011/03/24].

Received: April 21, 2011

Revised: May 19, 2011

Accepted: May 19, 2011

(C) David B. Smith; Licensee Bentham Open.

This is an open access article licensed under the terms of the Creative Commons Attribution Non-Commercial License (http://creativecommons.org/licenses/by-nc/3.0/) which permits unrestricted, non-commercial use, distribution and reproduction in any medium, provided the work is properly cited. 